\title{
Nucleic Acid Homologies of Some Vancomycin-Resistant Leuconostocs and Description of Leuconostoc citreum sp. nov. and Leuconostoc pseudomesenteroides sp. nov.
}

\author{
JOHN A. E. FARROW, ${ }^{1}$ RICHARD R. FACKLAM, ${ }^{2}$ AND MATTHEW D. COLLINS ${ }^{1 *}$ \\ Department of Microbiology, Agricultural Food Research Council Institute of Food Research, Reading Laboratory, \\ Shinfield, Reading RG2 9AT, United Kingdom, ${ }^{1}$ and Centers for Disease Control, Atlanta, Georgia $30333^{2}$
}

\begin{abstract}
Deoxyribonucleic acid-deoxyribonucleic acid hybridizations were performed with 31 vancomycin-resistant gram-positive cocci isolated from human sources. A total of 14 human isolates exhibited high levels of homology with Leuconostoc mesenteroides subsp. mesenteroides $\mathrm{NCDO} 523^{\mathrm{T}}$ ( $\mathrm{T}=$ type strain), whereas 2 strains were genetically closely related to Leuconostoc lactis NCDO $533^{\mathrm{T}}$. Nine human isolates formed a tight homology group with an atypical $L$. mesenteroides strain (strain NCIB 3351), whereas two isolates (together with four strains from food) were found to be highly related to Leuconostoc sp. strain NCDO $768^{\mathrm{T}}$. Two new species, Leuconostoc citreum and Leuconostoc pseudomesenteroides, are proposed for these homology groups. The type strains of $L$. citreum and $L$. pseudomesenteroides are strains NCDO 1837 and NCDO 768, respectively.
\end{abstract}

Vancomycin is a glycopeptide antibiotic that is used as a therapeutic agent for gram-positive coccal infections. Recently, there have been a number of reports of vancomycinresistant "leuconostocs"' from clinical sources $(1-3,10,11)$. Leuconostocs are normally found living in association with plant material and are common in dairy and other food products. Leuconostocs are generally considered nonpathogenic for humans and animals (6). The assignment of vancomycin-resistant clinical isolates to the genus Leuconostoc is based entirely on simple morphological and physiological tests. However, it is recognized that it is extremely difficult to reliably distinguish leuconostocs from some gram-positive cocci and certain lactobacilli (6). For example, the separation of leuconostocs from some heterofermentative lactobacilli (e.g., Lactobacillus confusus and Lactobacillus viridescens) is often based solely on Gram-stained films from broth cultures (leuconostocs are more coccoid than rodlike, and lactobacilli are more rodlike than coccoid). To our knowledge there has been no definitive identification of leuconostocs from human sources by using modern molecular techniques. Therefore, in this study 31 vancomycinresistant cocci isolated from clinical sources were examined by using deoxyribonucleic acid (DNA)-DNA molecular hybridization in an attempt to determine their generic and specific status.

\section{MATERIALS AND METHODS}

Strains. Details of the test strains and their sources are given in Table 1.

DNA base composition and DNA-DNA hybridization. Cells were grown in MRS broth at $25^{\circ} \mathrm{C}$ and were harvested in late exponential phase by centrifugation. DNA was prepared by using a modification (4) of the method of Garvie (5). DNA base composition was estimated by thermal denaturation in standard saline citrate, using DNA from Escherichia coli K-12 NCDO 1984 (guanine-plus-cytosine [G+C] content, $51.5 \mathrm{~mol} \%$ ) as the standard. DNA-DNA hybridizations were performed under optimum conditions $\left(25^{\circ} \mathrm{C}\right.$ below the melting temperature) by using the membrane filter method described previously (7). The washing procedure of Garvie (5) was modified to include one wash consisting of $10 \mathrm{ml}$ of 0.2

\footnotetext{
* Corresponding author.
}

$X$ standard saline citrate per filter to reduce nonspecific binding.

Biochemical tests. Biochemical tests were performed by using the API 50CH and API 10E systems (API-Biomerieux Ltd., Basingtoke, United Kingdom) according to the instructions of the manufacturer. Test preparations were incubated at $30^{\circ} \mathrm{C}$, and readings were made after 24 and $48 \mathrm{~h}$. Pigment production was observed in wet packed cells harvested from MRS broth by centrifugation.

\section{RESULTS AND DISCUSSION}

The DNA base compositions of the test strains are shown in Table 1. The clinical isolates had $\mathrm{G}+\mathrm{C}$ contents which ranged from ca. 36 to $43 \mathrm{~mol} \%$. These values are within the accepted $\mathrm{G}+\mathrm{C}$ content range (ca. 37 to $45 \mathrm{~mol} \%$ ) for the genus Leuconostoc $(8,9)$. The results of the DNA-DNA hybridization experiments are shown in Table 2 . The clinical isolates fell into four groups on the basis of DNA hybridization results. A total of 14 human isolates exhibited high homology levels (ca. 60 to 100\%) with the type strain of Leuconostoc mesenteroides subsp. mesenteroides (strain NCDO 523), whereas 2 strains were found to be genomically closely related to Leuconostoc lactis $\mathrm{NCDO} 533^{\mathrm{T}}$ ( $\mathrm{T}=$ type strain). Nine of the clinical strains formed a relatively homogeneous group which showed a high degree of relatedness (ca. 80 to $100 \%$ ) with an atypical Leuconostoc mesenteroides strain (strain NCIB 3351). Of the remaining human isolates, two strains were found to be closely related to Leuconostoc sp. strain NCDO $768^{\mathrm{T}}$. Four other strains isolated from food and designated Leuconostoc mesenteroides were also related to this group. Members of the strain NCIB 3351 and NCDO $768^{\mathrm{T}}$ homology groups were genetically distinct from each other and from all other Leuconostoc species. If $70 \%$ homology is taken as the border line or guideline for species differentiation, the DNA results clearly demonstrate that the two homology groups warrant separate specific status. The name Leuconostoc citreum sp. nov. is proposed for strains belonging to the strain NCIB 3351 group, and the name Leuconostoc pseudomesenteroides sp. nov. is proposed for the strain NCDO $768^{\mathrm{T}}$ group. The differential biochemical characteristics of Leuconostoc citreum, Leuconostoc pseudomesenteroides, and other Leuconostoc species are shown in Table 3. Leuconostoc citreum 
TABLE 1. Strain histories and DNA base compositions of the test strains

\begin{tabular}{|c|c|c|}
\hline Strain & Source and/or comments & $\begin{array}{l}\mathrm{G}+\mathrm{C} \\
\text { conten } \\
\text { (mol\% }\end{array}$ \\
\hline \multicolumn{3}{|c|}{ Leuconostoc citreum } \\
\hline NCDO $1837^{\mathrm{T}}$ & $\begin{array}{l}\text { Honey dew of rye ear; received as } \\
\text { Leuconostoc mesenteroides }\end{array}$ & 39.8 \\
\hline NCIB 3351 & $\begin{array}{l}\text { Canned tomatoes; received as } \mathrm{Leu}^{-} \\
\text {conostoc mesenteroides }\end{array}$ & 40.3 \\
\hline $1562-84$ & Blood & 39.4 \\
\hline $71-86$ & Blood & 38.0 \\
\hline $192-86$ & Blood & 39.4 \\
\hline $1352-86$ & Blood & 39.5 \\
\hline $2985-86$ & Blood & $\mathrm{ND}^{a}$ \\
\hline $3228-86$ & Blood, cerebrospinal fluid & 40.3 \\
\hline $133-87$ & Blood & 38.6 \\
\hline $1363-87$ & Medical source unknown & ND \\
\hline $2028-87$ & Blood & 39.2 \\
\hline \multicolumn{3}{|c|}{ Leuconostoc lactis } \\
\hline NCDO $533^{\mathrm{T}}$ & ATCC 19256, DSM 20202 & 44.7 \\
\hline NCDO 2037 & Received from T. Cogan & 43.1 \\
\hline $644-88$ & Blood & ND \\
\hline $606-88$ & Blood & ND \\
\hline \multicolumn{3}{|c|}{$\begin{array}{l}\text { Leuconostoc mesen- } \\
\text { teroides subsp. } \\
\text { cremoris (pheno- } \\
\text { type) }\end{array}$} \\
\hline NCDO $543^{\mathrm{T}}$ & $\begin{array}{l}\text { Dried starter powder; ATCC } 19254 \text {, } \\
\text { DSM } 20346\end{array}$ & 38.7 \\
\hline NCDO 183 & $\begin{array}{l}\text { Received as Leuconostoc dextrani- } \\
\quad \text { cum }\end{array}$ & 32.3 \\
\hline NCDO 769 & Swedish fermented milk & 37.8 \\
\hline NCDO 812 & $\begin{array}{l}\text { Received as Leuconostoc dextrani- } \\
\text { cum ATCC 8082; NCIB } 8189\end{array}$ & 38.0 \\
\hline NCDO 828 & The Netherlands & 39.3 \\
\hline NCDO 829 & The Netherlands & 37.8 \\
\hline NCDO 887 & $\begin{array}{l}\text { Received as Leuconostoc dextrani- } \\
\text { cum }\end{array}$ & 36.4 \\
\hline NCDO 1226 & Cheese starter & 38.8 \\
\hline NCDO 1388 & Received from P. R. Elliker & 38.8 \\
\hline NCDO 1589 & $\begin{array}{l}\text { Sauerkraut; received as Leuconos- } \\
\text { toc dextranicum }\end{array}$ & 37.1 \\
\hline \multicolumn{3}{|c|}{$\begin{array}{l}\text { Leuconostoc mesen- } \\
\quad \text { teroides subsp. } \\
\text { mesenteroides } \\
\text { (phenotype) }\end{array}$} \\
\hline NCDO $523^{\mathrm{T}}$ & Olives; ATCC 8293, DSM 20343 & 37.9 \\
\hline NCDO 520 & Silage & 35.2 \\
\hline NCDO 527 & Leuconostoc sp. & 36.7 \\
\hline NCDO $529^{\mathrm{T}}$ & $\begin{array}{l}\text { Leuconostoc dextranicum (type } \\
\text { strain) }\end{array}$ & 38.5 \\
\hline NCDO 540 & Produces dextran & 35.6 \\
\hline NCDO 547 & Raw milk & 34.0 \\
\hline NCDO 551 & $\begin{array}{l}\text { Fermenting beans; received as Leu- } \\
\text { conostoc dextranicum; ATCC } \\
8086, \text { NCIB } 8029\end{array}$ & 35.9 \\
\hline NCDO 797 & ATCC 9135, DSM 20241 & 36.4 \\
\hline NCDO 800 & $\begin{array}{l}\text { Slimy sugar syrup; received as Leu- } \\
\text { conostoc dextranicum }\end{array}$ & 34.1 \\
\hline NCDO 861 & $\begin{array}{l}\text { Cheese, The Netherlands; received } \\
\text { as Leuconostoc dextrancium }\end{array}$ & 37.3 \\
\hline NCDO 877 & Received from J. Naylor & 38.0 \\
\hline NCDO 888 & Received from J. Naylor & 37.2 \\
\hline NCDO 2028 & Beetroot silage & 37.3 \\
\hline $1190-77$ & Wound & 39.1 \\
\hline $738-78$ & Blood & 36.5 \\
\hline $872-78$ & Cerebrospinal fluid & 37.0 \\
\hline $1304-80$ & Blood & ND \\
\hline $1305-80$ & Blood & ND \\
\hline
\end{tabular}

TABLE $1-$ Continued

\begin{tabular}{|c|c|c|}
\hline Strain & Source and/or comments & $\begin{array}{l}\mathrm{G}+\mathrm{C} \\
\text { content } \\
(\mathrm{mol} \%)\end{array}$ \\
\hline $1007-81$ & Wound & 36.9 \\
\hline $1008-81$ & Medical, unknown & 37.0 \\
\hline $1055-82$ & Urine & ND \\
\hline $1591-82$ & Blood & 37.5 \\
\hline $1336-83$ & Blood & 37.5 \\
\hline $1661-85$ & Blood & 37.5 \\
\hline $225-86$ & Blood & ND \\
\hline $2906-87$ & Blood & 36.3 \\
\hline $128-88$ & Blood & 36.7 \\
\hline SML 41 & Meat; received from G. Shaw & 36.7 \\
\hline SML 50 & Meat; received from G. Shaw & 37.1 \\
\hline \multicolumn{3}{|c|}{$\begin{array}{l}\text { Leuconostoc para- } \\
\text { mesenteroides }\end{array}$} \\
\hline NCDO $803^{\mathrm{T}}$ & ATCC 33313, DSM 20288 & 39.3 \\
\hline NCDO 871 & Silage & 38.6 \\
\hline \multicolumn{3}{|c|}{$\begin{array}{l}\text { Leuconostoc } \\
\text { pseudomesen- } \\
\text { teroides }\end{array}$} \\
\hline NCDO $768^{\mathrm{T}}$ & $\begin{array}{l}\text { Received as Leuconostoc mesen- } \\
\quad \text { teroides ATCC 12991, DSM } 20193\end{array}$ & 39.3 \\
\hline NCDO 882 & Received from J. Naylor & 38.1 \\
\hline NCDO 1590 & $\begin{array}{l}\text { Received as Leuconostoc dextrani- } \\
\text { cum NCIB } 3740\end{array}$ & 40.6 \\
\hline NCDO 2053 & Starter, Switzerland & 38.6 \\
\hline NCDO 2069 & $\begin{array}{l}\text { Received as Leuconostoc mesen- } \\
\text { teroides NRRL B1416 }\end{array}$ & 40.8 \\
\hline $2187-81$ & Blood & 40.0 \\
\hline $1296-87$ & Blood & 38.7 \\
\hline \multicolumn{3}{|c|}{ Leuconostoc species } \\
\hline NCDO 1562 & Canker in hardwood & 35.9 \\
\hline $780-86$ & Lung & 38.0 \\
\hline $1882-86$ & Blood & 36.9 \\
\hline $2325-87$ & Blood & 42.5 \\
\hline $2850-87$ & Blood & ND \\
\hline $\mathrm{H} 18$ & Meat; received from G. Shaw & 37.8 \\
\hline L19 & Meat; received from $G$. Shaw & 38.6 \\
\hline $1 \mathrm{~A} 21$ & Meat; received from $G$. Shaw & 37.1 \\
\hline
\end{tabular}

${ }^{a} \mathrm{ND}$, Not determined.

produces a lemon yellow pigment and may be readily distinguished from Leuconostoc mesenteroides subsp. mesenteroides and Leuconostoc pseudomesenteroides because it does not produce acid from ribose. Strains of Leuconostoc citreum are also $\alpha$ - and $\beta$-D-galactosidase and $\beta$-Dxylosidase negative, whereas Leuconostoc mesenteroides subsp. mesenteroides and Leuconostoc pseudomesenteroides give positive reactions. In contrast, differentiation between Leuconostoc mesenteroides subsp. mesenteroides and Leuconostoc pseudomesenteroides is difficult. Leuconostoc mesenteroides subsp. mesenteroides produces acid from mannitol but not from starch after 7 days of incubation, whereas Leuconostoc pseudomesenteroides strains give the opposite reactions. In addition, Leuconostoc pseudomesenteroides is L-isoleucine arylamidase and L-proline arylamidase negative. Leuconostoc citreum and Leuconostoc pseudomesenteroides are easily distinguished from all other Leuconostoc species (Table 3).

Description of Leuconostoc citreum sp. nov. Leuconostoc citreum (cit're.um. M.L.adj. citreum, lemon colored). Spherical or lenticular cells (ca. 0.5 to 0.7 by 0.7 to $1.2 \mu \mathrm{m}$ ) that occur usually in pairs or short chains. Gram positive. Nonmotile. Most strains produce a lemon yellow pigment. Grows at 10 and $30^{\circ} \mathrm{C}$; some strains grow at $37^{\circ} \mathrm{C}$ but not at $40^{\circ} \mathrm{C}$. Acid is produced from $N$-acetylglucosamine, esculin, 
TABLE 2. DNA-DNA homologies of Leuconostoc species

\begin{tabular}{|c|c|c|c|c|c|c|}
\hline \multirow{3}{*}{ Strain } & \multicolumn{6}{|c|}{$\%$ Homology with $\left[{ }^{3} \mathrm{H}\right] \mathrm{DNA}$ from: } \\
\hline & \multirow{2}{*}{$\begin{array}{l}\text { Leuconostoc } \\
\text { citreum } \\
\text { NCIB } 3351\end{array}$} & \multirow{2}{*}{$\begin{array}{l}\text { Leuconostoc } \\
\text { lactis NCDO } \\
\quad 533^{\mathrm{T}}\end{array}$} & \multicolumn{2}{|c|}{$\begin{array}{l}\text { Leuconostoc mesenteroides } \\
\text { subsp. mesenteroides }\end{array}$} & \multirow{2}{*}{$\begin{array}{c}\text { Leuconostoc } \\
\text { pseudomesenteroides } \\
\text { NCDO } 768^{\mathrm{T}}\end{array}$} & \multirow{2}{*}{$\begin{array}{l}\text { Leuconostoc } \\
\text { paramesenteroides } \\
\text { NCDO } 803^{\mathrm{T}}\end{array}$} \\
\hline & & & NCDO $523^{\mathrm{T}}$ & NCDO 520 & & \\
\hline \multicolumn{7}{|l|}{ Leuconostoc citreum } \\
\hline NCDO $1837^{\mathrm{T}}$ & 91 & & 21 & & 27 & \\
\hline NCIB 3351 & 100 & 23 & 23 & 25 & 38 & 10 \\
\hline $1562-84$ & 101 & & 26 & & 38 & 16 \\
\hline $71-86$ & 102 & & 28 & & 34 & \\
\hline $192-86$ & 104 & & 25 & & 45 & 20 \\
\hline $1352-86$ & 100 & & 22 & & 33 & 16 \\
\hline $2985-86$ & 81 & & 23 & & 30 & 16 \\
\hline $3228-86$ & 100 & & 30 & & 54 & 17 \\
\hline $133-87$ & 100 & & 27 & & 47 & 20 \\
\hline $1363-87$ & 94 & & 29 & & 37 & 16 \\
\hline 2028-87 & 102 & & 26 & & 56 & 17 \\
\hline \multicolumn{7}{|l|}{ Leuconostoc lactis } \\
\hline NCDO $533^{\mathrm{T}}$ & & 100 & & 29 & & 12 \\
\hline NCDO 2037 & 52 & 81 & 21 & 41 & 28 & \\
\hline $644-88$ & 52 & 99 & 24 & 44 & 29 & 14 \\
\hline $606-88$ & 53 & 97 & 16 & & 28 & \\
\hline \multicolumn{7}{|c|}{$\begin{array}{l}\text { Leuconostoc mesenteroides subsp. } \\
\text { mesenteroides (phenotype) }\end{array}$} \\
\hline NCDO $523^{\mathrm{T}}$ & 27 & 7 & 100 & 62 & 43 & 14 \\
\hline NCDO 527 & & & 77 & & 45 & \\
\hline NCDO 540 & & & 108 & & 52 & \\
\hline NCDO 547 & & & 75 & & 24 & \\
\hline NCDO 551 & & & 94 & & 24 & \\
\hline NCDO 797 & & & 79 & & 35 & \\
\hline NCDO 861 & & & 87 & & 34 & \\
\hline NCDO 877 & & & 92 & & 62 & \\
\hline NCDO 888 & & & 83 & & 63 & \\
\hline NCDO 2028 & & & 86 & & 43 & \\
\hline $1190-77$ & & & 73 & & 45 & \\
\hline $738-78$ & & & 94 & & 38 & \\
\hline $1304-80$ & & & 92 & & 39 & \\
\hline $1305-80$ & & & 80 & & 33 & \\
\hline $1007-81$ & & & 76 & & 53 & \\
\hline $1008-81$ & & & 85 & & 43 & \\
\hline $1591-82$ & & & 75 & & 43 & \\
\hline $128-87$ & & & 75 & & 36 & \\
\hline $2906-87$ & & & 82 & & 38 & \\
\hline SML 41 & & & 86 & & 36 & \\
\hline NCDO 520 & 40 & 12 & 55 & 100 & 33 & \\
\hline NCDO 800 & 48 & & 62 & 73 & 29 & \\
\hline $865-78$ & 49 & 13 & 60 & 117 & 35 & 14 \\
\hline $872-78$ & 47 & 15 & 58 & 97 & 29 & \\
\hline $1055-82$ & 56 & & 66 & 110 & 50 & 16 \\
\hline $1336-83$ & 53 & & 70 & 100 & 44 & \\
\hline $1661-85$ & & & 69 & 106 & 44 & \\
\hline SML 50 & 52 & & 60 & 111 & 24 & \\
\hline \multicolumn{7}{|c|}{$\begin{array}{l}\text { Leuconostoc mesenteroides subsp. } \\
\text { cremoris (phenotype) }\end{array}$} \\
\hline NCDO $534^{\mathrm{T}}$ & 38 & 17 & 66 & 98 & 33 & \\
\hline NCDO 183 & 45 & & & 82 & 27 & \\
\hline NCDO 887 & 45 & 14 & & 77 & 27 & 10 \\
\hline NCDO 1226 & 48 & & 58 & 91 & 31 & \\
\hline NCDO 1388 & & & 72 & 79 & 30 & \\
\hline NCDO 1589 & & & 93 & & 37 & \\
\hline NCDO 529 & 54 & & 100 & & 39 & \\
\hline NCDO 769 & & & 101 & & 49 & \\
\hline NCDO 812 & & & 91 & & 28 & \\
\hline NCDO 828 & & & 106 & & 32 & \\
\hline NCDO 829 & & & 90 & & 50 & \\
\hline \multicolumn{7}{|c|}{ Leuconostoc pseudomesenteroides } \\
\hline NCDO $768^{\mathrm{T}}$ & 51 & 13 & 18 & 28 & 100 & 9 \\
\hline NCDO 882 & 44 & & 23 & & 90 & \\
\hline
\end{tabular}


TABLE 2-Continued

\begin{tabular}{|c|c|c|c|c|c|c|}
\hline \multirow{3}{*}{ Strain } & \multicolumn{6}{|c|}{$\%$ Homology with $\left[{ }^{3} \mathrm{H}\right] \mathrm{DNA}$ from: } \\
\hline & \multirow{2}{*}{$\begin{array}{l}\text { Leuconostoc } \\
\text { citreum } \\
\text { NCIB } 3351\end{array}$} & \multirow{2}{*}{$\begin{array}{l}\text { Leuconostoc } \\
\text { lactis NCDO } \\
\qquad 533^{\mathrm{T}}\end{array}$} & \multicolumn{2}{|c|}{$\begin{array}{l}\text { Leuconostoc mesenteroides } \\
\text { subsp. mesenteroides }\end{array}$} & \multirow{2}{*}{$\begin{array}{c}\text { Leuconostoc } \\
\text { pseudmesenteroides } \\
\text { NCDO } 768^{\mathrm{T}}\end{array}$} & \multirow{2}{*}{$\begin{array}{c}\text { Leuconostoc } \\
\text { paramesenteroide } \\
\text { NCDO } 803^{\mathrm{T}}\end{array}$} \\
\hline & & & NCDO $523^{\mathrm{T}}$ & NCDO 520 & & \\
\hline NCDO 1590 & 55 & & 33 & & 100 & \\
\hline NCDO 2053 & & & 38 & & 100 & 16 \\
\hline NCDO 2069 & 51 & & 31 & 50 & 70 & \\
\hline $2187-81$ & & & 46 & & 100 & \\
\hline $1596-87$ & & & 32 & & 103 & \\
\hline \multicolumn{7}{|c|}{ Leuconostoc paramesenteroides } \\
\hline NCDO $803^{\mathrm{T}}$ & 22 & 8 & 9 & 13 & 14 & 100 \\
\hline NCDO 871 & 31 & & 10 & & 30 & 100 \\
\hline \multicolumn{7}{|l|}{ Leuconostoc sp. } \\
\hline 1A 21 & 46 & & 24 & & 53 & 11 \\
\hline $\mathrm{H} 18$ & 52 & 17 & 20 & 24 & 43 & 17 \\
\hline L19 & 50 & 19 & 22 & 32 & 37 & 14 \\
\hline NCDO 1562 & 44 & 10 & 31 & 42 & 31 & \\
\hline $780-86$ & 40 & & 10 & 20 & 16 & \\
\hline $1882-86$ & 54 & & 43 & 36 & 51 & 20 \\
\hline $2325-87$ & 34 & & 26 & 30 & 24 & \\
\hline $2850-87$ & 49 & & & 37 & & 19 \\
\hline
\end{tabular}

D-fructose, glucose, D-mannose, maltose, $\alpha$-methyl-D-glucoside, sucrose, trehalose, and D-turanose. The vast majority of strains produce acid from amygdalin, arbutin, Larabinose, cellobiose, $\beta$-gentiobiose, gluconate, D-mannitol, and salicin. A few strains produce acid from 2-keto-glu- conate, galactose, and D-xylose. Acid is not produced from adonitol, D-arabitol, L-arabitol, D-arabinose, dulcitol, erythritol, D-fucose, L-fucose, glycerol, glycogen, inositol, inulin, 5-keto-gluconate, lactose, D-lyxose, melezitose, melibiose, $\alpha$-methyl-D-mannoside, $\beta$-methyl-xyloside, raffinose, ribose,

TABLE 3. Differential characteristics of Leuconostoc species

\begin{tabular}{|c|c|c|c|c|c|c|}
\hline Characteristic & $\begin{array}{l}\text { Leuconostoc } \\
\quad \text { citreum } \\
(n=11)^{a}\end{array}$ & $\begin{array}{l}\text { Leuconostoc } \\
\quad \text { lactis } \\
\quad(n=4)\end{array}$ & $\begin{array}{l}\text { Leuconostoc } \\
\text { mesenteroides subsp. } \\
\text { mesenteroides } \\
(n=30)\end{array}$ & $\begin{array}{l}\text { Leuconostoc } \\
\text { mesenteroides } \\
\text { subsp. cremoris } \\
\quad(n=14)\end{array}$ & $\begin{array}{c}\text { Leuconostoc } \\
\text { paramesenteroides } \\
(n=3)\end{array}$ & $\begin{array}{c}\text { Leuconostoc } \\
\text { pseudomesenteroides } \\
(n=7)\end{array}$ \\
\hline \multicolumn{7}{|l|}{ Acid produced from ${ }^{b}$ : } \\
\hline L-Arabinose & $91^{c}$ & 25 & 83 & 0 & 100 & 71 \\
\hline Ribose & 0 & 0 & 84 & 28 & 100 & 100 \\
\hline D-Xylose & 18 & 0 & 96 & 29 & 0 & 100 \\
\hline D-Galactose & 18 & 100 & 87 & 86 & 100 & 86 \\
\hline D-Fructose & 100 & 100 & 100 & 43 & 100 & 100 \\
\hline D-Mannose & 100 & 100 & 100 & 43 & 100 & 100 \\
\hline D-Mannitol & 55 & 0 & $18(97)^{d}$ & $7(14)$ & $0(100)$ & $0(14)$ \\
\hline$\alpha$-Methyl-D-glucoside & 100 & 0 & 94 & 21 & 100 & 86 \\
\hline Amygdalin & 82 & 0 & 60 & 0 & 0 & 29 \\
\hline Arbutin & 91 & 0 & 66 & 0 & 0 & 14 \\
\hline Esculin & 100 & 0 & 97 & 7 & 33 & 57 \\
\hline Salicin & 91 & 0 & 93 & 0 & 33 & 43 \\
\hline Cellobiose & 91 & 0 & 73 & 0 & 33 & 71 \\
\hline Maltose & 100 & 100 & 100 & 21 & 100 & 100 \\
\hline Lactose & 0 & 100 & 53 & 65 & 0 & 58 \\
\hline Melibiose & 9 & 75 & 97 & 29 & 100 & 86 \\
\hline Sucrose & 100 & 100 & 100 & 50 & 100 & 71 \\
\hline Trehalose & 100 & 50 & 100 & 43 & 100 & 100 \\
\hline Raffinose & 0 & 75 & 77 & 21 & 33 & 86 \\
\hline Starch & 0 & 0 & 0 & 0 & 0 & $14(76)$ \\
\hline$\beta$-Gentiobiose & 91 & 0 & 70 & 7 & 33 & 58 \\
\hline D-Turanose & 100 & 0 & 87 & 7 & 100 & 71 \\
\hline Gluconate & 91 & 0 & 57 & 7 & 100 & 57 \\
\hline 2-Keto-gluconate & $45(63)$ & 0 & 3 & 0 & 33 & $14(43)$ \\
\hline 5-Keto-gluconate & 0 & 0 & $0(12)$ & 0 & 0 & $14(43)$ \\
\hline Lemon yellow pigment $t^{e}$ & 91 & 0 & 7 & 14 & 0 & 14 \\
\hline
\end{tabular}

$a_{n} n$ is the number of strains tested.

${ }^{b}$ Tests were performed by using the API $50 \mathrm{CH}$ system; readings were determined at $48 \mathrm{~h}$.

c Percentage of strains positive.

${ }^{d}$ Values in parentheses refer to readings at 7 days

e Pigment detected in centrifuged cell pellet from MRS broth. 
L-rhamnose, sorbitol, L-sorbose, starch, D-tagatose, xylitol, or L-xylose. Arginine dihydrolase, $\beta$-D-xylosidase, and urease negative. The $\mathrm{G}+\mathrm{C}$ content of the DNA ranges from 38.0 to $40.3 \mathrm{~mol} \%$ (thermal denaturation method). Isolated from food and clinical sources. The type strain is strain NCDO 1837. In most respects the description of the type strain corresponds to the description of the species. The type strain produces acid from amygdalin, L-arabinose, arbutin, cellobiose, $\beta$-gentiobiose, gluconate, mannitol, and salicin. Acid is not produced from melibiose, raffinose, or D-xylose.

Description of Leuconostoc pseudomesenteroides sp. nov. Leuconostoc pseudomesenteroides (pseu.do.me.sen.ter. oi'des. Gr.adj. pseudes, false; Gr.n. mesenterium, the mesentery; Gr.n. oides, form, shape; M.L.adj. pseudomesenteroides, not the true mesenteroides). Spherical or lenticular cells (ca. 0.5 to 0.7 by 0.7 to $1.2 \mu \mathrm{m}$ ) that occur usually in pairs or short chains. Gram positive. Nonmotile. Most strains are nonpigmented. Grows at 10 and $37^{\circ} \mathrm{C}$. Acid is produced from $N$-acetylglucosamine, D-fructose, D-glucose, maltose, D-mannose, melibiose, $\alpha$-methyl-D-glucoside, raffinose, ribose, trehalose, and D-xylose. Most strains produce acid from L-arabinose, cellobiose, esculin, galactose, $\beta$ gentiobiose, lactose, sucrose, and D-turanose. A few strains produce acid from amygdalin, arbutin, 2-keto-gluconate, 5-keto-gluconate, and mannitol (after 7 days). Acid is not produced from adonitol, D-arabinose, D-arabitol, L-arabitol, dulcitol, erythritol, D-fucose, L-fucose, glycerol, inositol, inulin, D-lyxose, melezitose, $\alpha$-methyl- $D$-mannoside, Lrhamnose, L-sorbose, sorbitol, D-tagatose, L-xylose, or $\mathrm{xy}$ litol. Arginine dehydrolase, urease, L-isoleucine arylamidase, and $\mathrm{L}$-proline arylamidase negative. The $\mathrm{G}+\mathrm{C}$ content of the DNA ranges from 38.1 to $40.8 \mathrm{~mol} \%$ (thermal denaturation method). Isolated from dairy sources, food, and clinical sources. The type strain is strain NCDO 768. In most respects the description of the type strain resembles the description of the species. The type strain produces acid from L-arabinose, cellobiose, 2-keto-gluconate, 5-keto-glu- conate, galactose, $\beta$-gentiobiose, gluconate, and lactose. Acid is not produced from amygdalin, arbutin, mannitol (after 7 days), salicin, or starch.

\section{LITERATURE CITED}

1. Colman, G., and A. Efstratiou. 1987. Vancomycin-resistant leuconostocs, lactobacilli and now pediococci. J. Hosp. Infect. 10:1-3.

2. Coovadia, Y. M., Z. Solwa, and J. Van Den Ende. 1987. Meningitis caused by vancomycin-resistant Leuconostoc sp. J. Clin. Microbiol. 25:1784-1785.

3. Coovadia, Y. M., Z. Solwa, and J. Van Den Ende. 1988. Potential pathogenicity of Leuconostoc. Lancet i:3-7.

4. Farrow, J. A. E., D. Jones, B. A. Phillips, and M. D. Collins. 1983. Taxonomic studies on some group D streptococci. J. Gen. Microbiol. 129:1423-1432.

5. Garvie, E. I. 1976. Hybridization between the deoxyribonucleic acids of some strains of heterofermentative lactic acid bacteria. Int. J. Syst. Bacteriol. 26:116-122.

6. Garvie, I. E. 1986. Genus Leuconostoc, p. 1071-1075. In P.H.A. Sneath (ed.), Bergey's manual of systematic bacteriology, vol. 2. The Williams \& Wilkins Co., Baltimore.

7. Garvie, E. I., J. A. E. Farrow, and B. A. Phillips. 1981. A taxonomic study of some strains of streptococci which grow at $10^{\circ} \mathrm{C}$ but not at $45^{\circ} \mathrm{C}$, including Streptococcus lactis and Streptococcus cremoris. Zentralbl. Bakteriol. Parasitenkd. Infektionskr. Hyg. Abt. 1 Orig. Reihe C 2:151-165.

8. Garvie, E. I., V. Zezula, and V. A. Hill. 1974. Guanine plus cytosine content of the deoxyribonucleic acids of the leuconostocs and some heterofermentative lactobacilli. Int. J. Syst. Bacteriol. 24:248-251.

9. Hontebyrie, M., and F. Gasser. 1977. Deoxyribonucleic acid homologies in the genus Leuconostoc. Int. J. Syst. Bacteriol. 27:9-14.

10. Horowitz, H. W., S. Handwerger, K. G. Van Horn, and G. P. Wormser. 1987. Leuconostoc, an emerging vancomycin-resistant pathogen. Lancet ii:1329-1330.

11. Isenberg, H. D., E. M. Vellozzi, J. Shapiro, and L. G. Rubin. 1988. Clinical laboratory challenges in the recognition of Leuconostoc spp. J. Clin. Microbiol. 26:479-483. 